\title{
Apps de entrega a domicilio en CDMX: estrategia restaurantera de ventas para sobrevivir a la pandemia
}

Food Delivery Apps in CDMX: Sales Strategy to Survive the Pandemic

\begin{abstract}
Aplicativos de entrega em domicílio no CDMX: estratégia de vendas de restaurantes para sobreviver à pandemia
\end{abstract}

\author{
Juan Manuel Sandoval Damián \\ Instituto Politécnico Nacional, México \\ jsandovald0900@alumno.ipn.mx \\ https://orcid.org/0000-0002-9048-025X \\ José Leonardo Serralde Coloapa \\ Instituto Politécnico Nacional, México \\ jserraldec1700@alumno.ipn.mx \\ https://orcid.org/0000-0003-3968-2160 \\ Elizabeth Acosta Gonzaga \\ Instituto Politécnico Nacional, México \\ eacostag@ipn.mx \\ https://orcid.org/0000-0001-5413-1063
}

\section{Resumen}

La industria restaurantera es uno de los sectores más productivos y representativos de la actividad turística en México, por el gran número de establecimientos que la componen. Debido al cierre de establecimientos a causa de la pandemia de COVID-19, se ha tenido que recurrir al uso de aplicaciones digitales, llamadas food delivery apps, las cuales habilitan la comunicación entre los restaurantes y sus clientes para entregar alimentos preparados a domicilio. Para describir el uso de estas aplicaciones por los restaurantes, se realizó una investigación de tipo cualitativa descriptiva que incluyó una entrevista a un grupo de MIPyMES restauranteras de dos alcaldías de la Ciudad de México. Los resultados muestran 


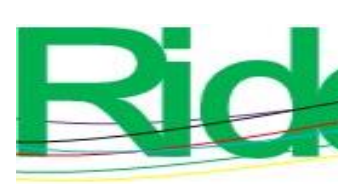

\section{Revista Iberoamericana para la Investigación y el Desarrollo Educativo ISSN 2007 - 7467}

dos restaurantes corriam risco de fechamento definitivo. Porém, devido ao uso de aplicativos de entrega de comida, os restaurantes conseguiram obter aumentos de vendas de $20 \%$ a $50 \%$, o que indica que os aplicativos digitais ajudaram as empresas a continuar operando e sobreviver à pandemia provocada pelo coronavírus de 2019.

Palavras-chave: COVID-19, aplicativos de entrega de comida, MPMEs, pandemia, restaurantes.

Fecha Recepción: Marzo 2021

Fecha Aceptación: Noviembre 2021

\section{Introducción}

El ser humano ha enfrentado diversos retos para garantizar la supervivencia de su especie. Uno de ellos, sin lugar a dudas, han sido las enfermedades contagiosas. Según la Organización Mundial de la Salud [OMS] (11 de octubre de 2018, 25 de septiembre de 2017, 16 de abril de 2019), México ha enfrentado, en los últimos años, tres brotes epidémicos importantes: la infección por Pseudomonas aeruginosas resistentes a los carbapenémicos en 2019, la infección por el virus del Zika en 2015 y el cólera en 2013. En 2019, se detectó por primera vez en China el coronavirus de tipo 2 causante del síndrome respiratorio agudo severo (SARS-CoV-2) que causa la enfermedad por coronavirus de 2019 (COVID-19) y desde entonces se ha expandido por todo el mundo a gran velocidad y ha causado grandes afectaciones a nivel global (Organización de la Naciones Unidas [ONU], 16 de marzo de 2020). Por supuesto, este virus era desconocido para la población mexicana antes de que comenzara el brote en China (Universidad Nacional Autónoma de México [UNAM], 2020).

El 11 de marzo, el director general de la OMS, durante una conferencia de prensa sobre la COVID-19, hablaba de 118000 casos en 114 países y 4291 personas fallecidas (OMS, 2020). En México, días antes a esta intervención, el 28 de febrero del 2020, ya se había detectado el primer caso de COVID-19, y comenzaba a hablarse a nivel nacional de las fases de transmisión del coronavirus (Miranda y Morales, 2020). De acuerdo con la Secretaría de Salud [SS] (12 de marzo de 2020), durante la fase 1 no era necesario suspender viajes nacionales o internacionales, cerrar puertos o fronteras.

Al igual que el resto de los países, México se enfrentó a una situación sin precedentes (Secretaría de Salud, 24 de marzo de 2020), mucho más severa a la vivida en el 2009, cuando la pandemia de influenza A H1N1, la cual, de acuerdo con el Centro Nacional de Programas Preventivos y Control de Enfermedades [CENAPRECE] (2018), requirió la participación de 


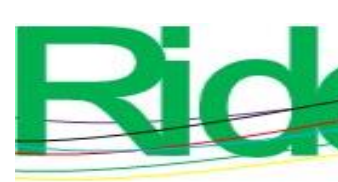

Revista Iberoamericana para la Investigación y el Desarrollo Educativo ISSN $2007-7467$

diversos organismos para la creación de planes y estrategias para contener la propagación del virus y minimizar el daño en los diversos sectores del país.

Para el 24 de marzo del 2020, se dio inicio a la fase 2 por COVID-19 (Secretaría de Salud, 24 de marzo de 2020), fase de dispersión comunitaria, por lo que el Gobierno de México implementó una serie de estrategias; comenzó con la contratación de personal médico, la mejora de infraestructura hospitalaria y la elaboración de planes estratégicos económicos para poder hacer frente al número de contagios que crecía de forma exponencial.

El 21 de abril del 2020 la Secretaría de Salud decretó el inició de la fase 3. La Jornada Nacional de Sana Distancia y la suspensión temporal de actividades no esenciales fueron parte de la estrategia para disminuir la propagación del virus en la población mexicana. No está de más hacer hincapié en el cierre de actividades económicas no esenciales para la supervivencia de la especie humana, esto en los sectores tanto público como privado a nivel nacional.

Con el inicio de la fase 3, la principal estrategia para disminuir la propagación del virus consistió en el cierre obligatorio de establecimientos comerciales, sobre todo aquellos que propiciaran reuniones o aglomeraciones, entre los que destacan teatros, cines, restaurantes, bares, cafeterías y otros (Konfío, 2020a). Esta situación representó diversos problemas en México. Una de las industrias más afectadas fue la restaurantera, de las más importantes para el país ya que representa, según el Instituto Nacional de Estadística y Geografía [INEGI] (2019), $1.8 \%$ del producto bruto total (PBT) y $1.9 \%$ del consumo intermedio, lo que a su vez representa $1.1 \%$ del producto interno bruto (PIB) total del país. También es importante recalcar que la industria restaurantera tenía empleadas en 2019 a un total de 1475981 personas, lo que le atribuía el segundo lugar en generación de empleo.

El cierre obligatorio afectó directamente a 2.14 millones de empleos, lo que generó una reducción de $90 \%$ en ventas y $30 \%$ de unidades económicas que han tenido que cerrar (Fernández, 26 de marzo de 2020).

La presente investigación analizó cómo el uso de aplicaciones tecnológicas de food delivery ha servido para mantener las ventas de las micro, pequeñas y medianas empresas (MIPyMES) restauranteras mexicanas y sobrevivir a la orden de cierre de negocios y confinamiento obligatorio. 

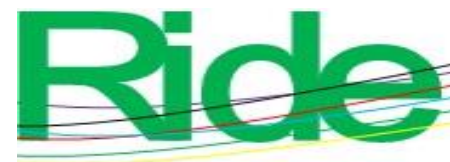

Revista Iberoamericana para la Investigación y el Desarrollo Educativo ISSN $2007-7467$

\section{Marco teórico}

Dos meses después de la declaración del Dr. Tedros Adhanom Ghebreyesus, director de la OMS (11 de marzo de 2020), de la COVID-19 como pandemia, el gobierno mexicano ordenó el cierre masivo de establecimientos comerciales, situación que afectó a diversas industrias y sectores económicos, entre los que destaca la industria restaurantera. Siguiendo a la Cámara Nacional de la Industria de Productos Alimenticios Condimentados [CANIRAC] (2015), en México existen 568866 unidades económicas pertenecientes a los servicios de alojamiento temporal y de preparación de alimentos y bebidas; de ese total, $97 \%$, correspondiente a 544937 unidades, pertenece al subsector de servicios de preparación de alimentos. Además, hay 515059 establecimientos que se dedican a la preparación de alimentos y bebidas alcohólicas y no alcohólicas. Cabe señalar que 58 de estas unidades económicas forman $4.5 \%$ de la tasa de crecimiento anual de establecimientos de la industria. La tabla 1 muestra la cantidad de unidades económicas por su actividad. 


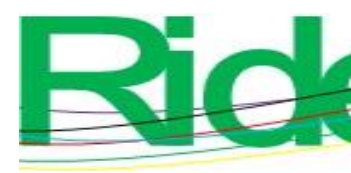

Revista Iberoamericana para la
Investigación y el Desarrollo Educativo
ISSN $2007-7467$

de forma urgente y se adaptaron al sistema al negocio. Sin embargo, habría que considerar que del total de los negocios en el país solo $10 \%$ ocupaban este servicio.

De acuerdo con lo que menciona González, quien también es presidente de Directores de Cadenas de Restaurantes, A.C. (DICARES), $70 \%$ de los restaurantes en México estaba en riesgo de desaparecer. De los más de 600000 restaurantes, $97 \%$ son microempresas, y de ese total, en ese momento, $90 \%$ se encontraba cerrado por la pandemia; únicamente $10 \%$ continuaba con operaciones, esto gracias a que habían establecido alianzas comerciales de entrega a domicilio, incluyendo el uso de las food delivery apps, sin embargo, esta estrategia únicamente generaba ventas por una cantidad que va de $10 \%$ a $15 \%$ (AMR, 24 de abril de 2020).

Cabe mencionar que diversas empresas dedicadas a las aplicaciones food delivery en México lanzaron comunicados en los que anunciaban apoyos a restaurantes, tal y como el pago diario, en lugar de hacerlo semanalmente, implementar los envíos gratis para pequeñas y medianas empresas (PyMES) y apoyar con el marketing para que la visibilidad de los restaurantes locales fuera de mayor alcance. Dichas acciones fueron de gran apoyo en su momento para un estimado de más de 30000 restaurantes (AMR, 24 de abril de 2020). Estas acciones coadyuvaron a que las MIPyMES del sector aminoraran la carga económica y evitaron el cierre permanente del establecimiento.

Las food delivery apps son conceptualizadas como el servicio que se encarga de entregar alimentos, pueden ser preparados o no, ordenados en línea (Statista, 2020). Asimismo, hay dos variantes: $a$ ) entrega de restaurantes a consumidor, siendo esta la principal en México, con un valor de mercado de 1077 millones de dólares en 2020, y b) entrega de plataforma a consumidor. También, según Statista (2020), los ingresos por esta actividad durante el 2020 en México superan los 1832 millones de dólares. Y se espera que estos ingresos tengan una tasa de crecimiento anual de $9.76 \%$ (Statista, 2020).

Actualmente, en el mercado existen diversas aplicaciones de entrega de comida. Las principales en México son Sin Delantal, Uber Eats, Rappi, Postmates, Cornershop y Mercadoni (Flores, 2018).

Y según datos de Escamilla (8 de abril de 2020), el uso de estas aplicaciones ha ido en aumento y alcanzará un valor de 1478 millones de dólares durante el 2021, debido al aumento de demanda y popularidad.

Galeano (2019) explica que Uber Eats (con un ticket promedio de 210 pesos), Rappi (180 pesos) y Sin Delantal (con un ticket promedio de 110 pesos), son las aplicaciones que 


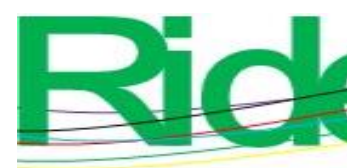

Revista Iberoamericana para la
Investigación y el Desarrollo Educativo
ISSN $2007-7467$

más se usan entre los mexicanos: un promedio de cuatro veces por mes con un rango de gasto de acuerdo con la edad. Las personas con una edad menor de 25 años gastan en promedio un total de 140 pesos, las personas que se encuentran en un rango de edad mayor, de entre 25 y 34 años, presentan un consumo promedio de $\$ 180$, mientras que las personas con una edad superior a los 45 años de edad gastan en promedio $\$ 340$ cada vez que hacen uso de los servicios que ofrecen las aplicaciones.

Ahora bien, según Nabor (13 de mayo de 2020), el plan para reactivar la economía nacional incluía a mediados del año pasado que los restaurantes podrían volver a operar a partir del 15 de junio de 2020 y solo a un tercio de su capacidad, además de seguir medidas de higiene y distanciamiento social.

Si bien es un momento muy difícil para los microempresarios dueños de restaurantes, Giles (2020) y Konfío (2020b) señalan que este momento representa una oportunidad no solo para que los establecimientos sobrevivan, sino también para cambiar y evolucionar las paradigmas tradicionales sobre sus ventas y servicio que ofrecen actualmente.

Aunque las oportunidades de actuar son escasas y no exentas de barreras, los restaurantes que aún sobreviven plantean al comercio electrónico como una vía de escape al adoptar prácticas innovadoras como el uso de food delivery apps y las dark kitchens que ofrecen beneficios económicos para los restaurantes (Fernández, 2020).

Para ahondar más en el uso que han tenido las food delivery apps en los restaurantes, se hizo una revisión de la base de datos Web of Science, que incluyó el periodo de 1980 al 2020. Se encontró un total de 30 registros, de los cuales se delimitó, por cuestiones de espacio, a cuatro investigaciones que a continuación se reseñan.

La investigación realizada por He, Han, Cheng, Fan y Dong (2019) analizó la competitividad de los restaurantes dentro del creciente mercado del food delivery. En este estudio se analizaron tres factores y se descubrió que el criterio de los clientes tenía influencia sobre las decisiones de los restaurantes.

Asimismo, Cho, Bonn y Li (2019) estudiaron la evolución en la forma de comprar alimentos en China y cómo las aplicaciones de food delivery han aprovechado esta oportunidad para afianzarse en el mercado y producir ingresos propios para los establecimientos de alimentos y bebidas. Aquí se encontró que los hogares con una sola persona tienen preferencias sobre la calidad de entre las opciones de alimentos, el precio y la fiabilidad, y que los hogares que están compuestos por más de una persona tienen preferencias e intereses hacia el diseño, a la convivencia y a la fiabilidad. 


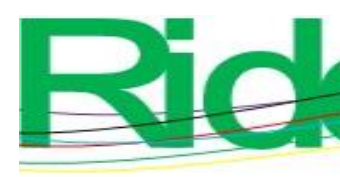

Revista Iberoamericana para la Investigación y el Desarrollo Educativo ISSN $2007-7467$

Por su parte, Chung, Choi y Choi (2017) observaron que cada vez es más fácil acceder al mercado de aplicaciones dedicadas al food delivery. En este trabajo, el objetivo fue analizar los efectos del riesgo percibido y los beneficios en las compras de alimentos por las aplicaciones en Seúl y Gyeonggi-do. También analizó lo que las empresas deben hacer para volverse competitivas en el mercado.

Por último, Kim, Tang and Bosselman (2019) analizaron la cocreación de valor por el servicio entregado de los restaurantes y cómo este influye en la fidelización y la lealtad de los clientes. Los investigadores proponen que la innovación que el cliente percibe es un elemento importante para predecir su comportamiento, lo cual, a su vez, influye en su satisfacción y lealtad.

\section{Materiales y métodos}

Dentro de la Ciudad de México, se eligieron las alcaldías Cuauhtémoc y Miguel Hidalgo, debido a que son zonas de alto flujo turístico, y también por la cantidad de restaurantes que se encuentran en operación. La investigación fue tipo cualitativa descriptiva, ya que el uso de este enfoque es apropiado cuando el fenómeno a estudiar está conformado por diversos factores, comportamientos y conceptos. Se busca comprender las situaciones tal y como existen, mediante la colecta de datos congruentes con el entorno y el fenómeno a estudiar (Hernández y Mendoza, 2018) y, de esta manera, obtener un acercamiento considerable a la problemática de la realidad social actual (Sarduy, 2007; Ugalde y Balbastre, 2013).

Los restaurantes elegidos se encontraban en operación únicamente bajo la modalidad de entrega a domicilio y haciendo uso de aplicaciones de food delivery para cumplir con la nueva modalidad de operación propuesta por el Gobierno de la Ciudad de México. Se adoptó una metodología de trabajo online para la aplicación de la entrevista estructurada. Esta forma de trabajar derivó del confinamiento voluntario y del cierre de establecimientos para ventas físicas que se vivía en todo el país. Se hizo uso de Facebook, WhatsApp y también de llamadas telefónicas y correos electrónicos. Estas herramientas se usaron para establecer contacto con los restaurantes participantes y poder entrevistarlos. Se garantizó el anonimato, el uso responsable y ético de los datos obtenidos.

Para el diseño de la entrevista no se eligió una lógica de variables, sino que se delimitó por temas principales (Yin, 2003): ventas de los establecimientos antes y después de la pandemia y uso de las aplicaciones de food delivery, incluyendo el tipo de plataforma y sus 


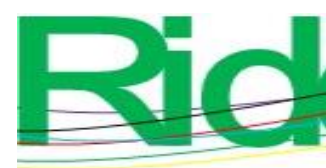

Revista Iberoamericana para la
Investigación y el Desarrollo Educativo
ISSN $2007-7467$

características. Por lo que la entrevista se dividió en dos secciones. La primera sección recopiló información sobre las ventas, y la segunda sección se centró en el uso de las apps. En esta sección también se preguntó directamente a los restauranteros si consideraban que podrían seguir operando bajo esta modalidad (ver anexo).

El muestreo fue por conveniencia, no probabilístico, con base en la accesibilidad y proximidad de los sujetos de estudio (Hernández y Mendoza, 2018). La selección y recolección de datos se realizó al inicio de la pandemia por COVID-19, al inicio del confinamiento y cierre obligatorio de establecimientos, por lo que pocos restaurantes se encontraban en operación. Se identificó, contactó y entrevistó a un grupo de restauranteros durante los meses de abril a junio del 2020 y se obtuvieron 14 entrevistas. Durante el análisis de los datos se detectó que varias de las respuestas incluían cifras, por lo que se graficaron los datos para su mejor entendimiento.

\section{Resultados}

El presente estudio analizó la opinión de diversos restauranteros de dos alcaldías de la Ciudad de México para conocer el impacto de la pandemia por COVID-19 en sus ventas al público, y mostró que el uso de aplicaciones de food delivery ha sido una herramienta muy útil para que las pudieran seguir en operación.

La primera sección de la entrevista recolectó datos sobre la disminución en las ventas que los restauranteros tuvieron después del anuncio de cierre obligatorio de los establecimientos y su operación únicamente por entrega a domicilio. Los datos se muestran en la figura 1.

Figura 1. Ventas (en porcentaje) de los restaurantes después del cierre obligatorio

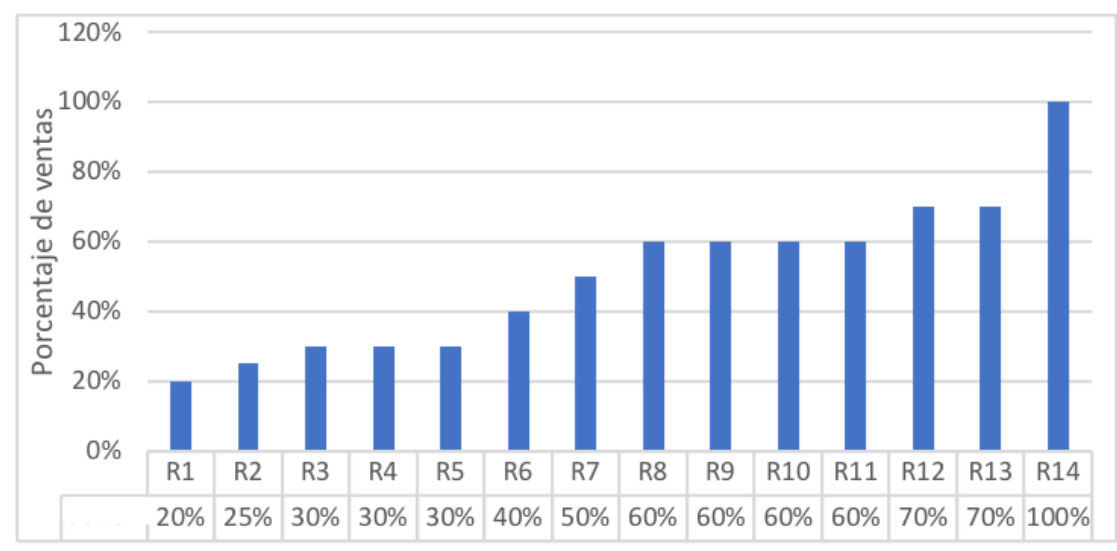

Fuente: Elaboración propia 

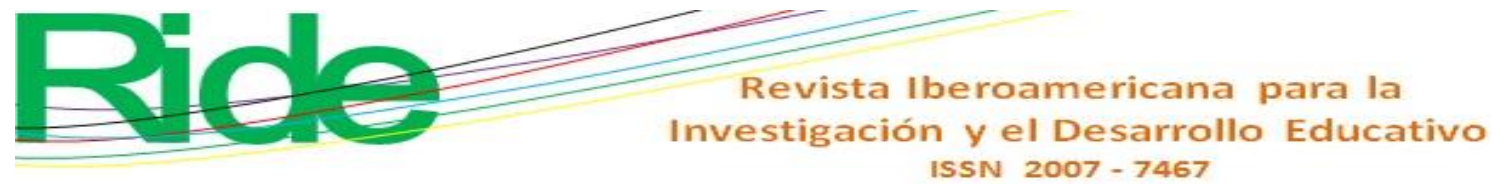

Del total de los restaurantes, siete de ellos sufrieron afectaciones que los llevaron a una disminución en sus ventas de $80 \%$ a $90 \%$.

En esa misma línea, 43 \% de los restaurantes entrevistados tuvieron una reducción de sus ventas entre $60 \%$ y $75 \%$. Y solo en un restaurante se observó una reducción en sus ventas menor de $20 \%$, esto debido a que ya usaba las food delivey apps como forma de trabajo. Por lo tanto, se observó que, después del cierre de restaurantes, las ventas se redujeron drásticamente, lo que puso en peligro la operación de la mayoría de los establecimientos.

En la figura 2 se muestra la manera en que el uso de aplicaciones food delivery influyó de forma positiva para que las MIPyMES restauranteras continuaran solventando gastos de operación, mantener al personal de trabajo, ya sea parcial o totalmente dentro de su nómina, y poder seguir laborando. Gracias a estas herramientas digitales, $29 \%$ de los establecimientos se encontraba sin riesgo de cerrar sus operaciones. Cabe mencionar que también influyeron diversos aspectos para que se encontraran fuera de peligro del cierre, como un alto posicionamiento en el mercado, precios accesibles y que se adaptaron a la capacidad de pago de los comensales habituales.

La mayoría de restaurantes no tenían estrategias alternas de operación para continuar trabajando. Algunos de ellos copiaban otras formas de trabajo, como la publicidad en las fachadas de los establecimientos; otros restaurantes ajustaron sus precios de venta, modificaron sus productos, sin embargo, esto no representó un beneficio significativo para la supervivencia de los establecimientos, lo cual se reflejó en los datos obtenidos, ya que $71 \%$ se encontraba en riesgo de cierre definitivo.

Figura 2. Porcentaje de restaurantes en riesgo de cerrar sus operaciones

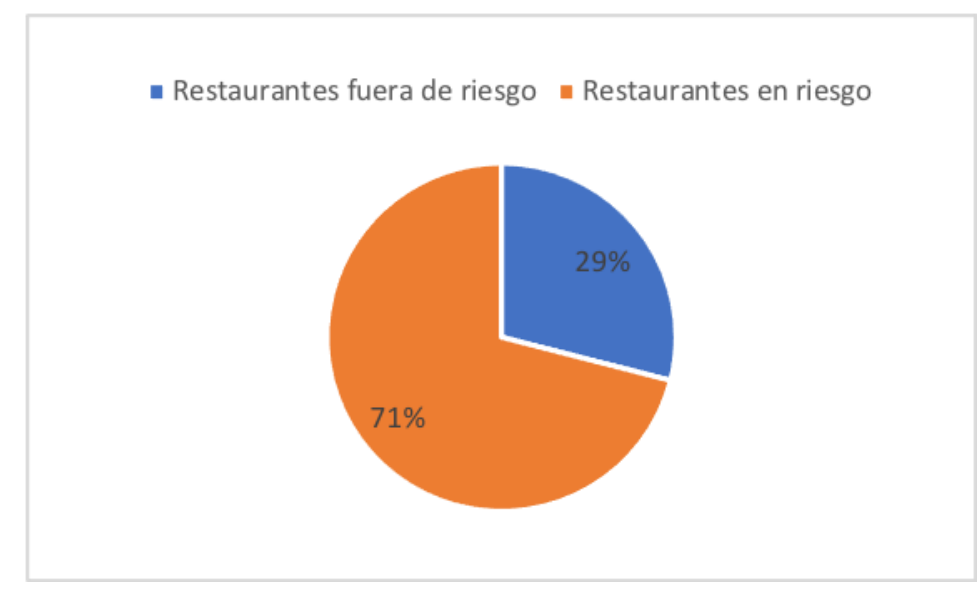

Fuente: Elaboración propia 


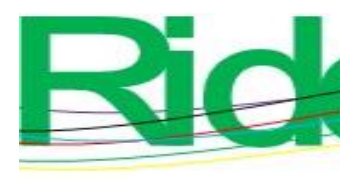

Revista Iberoamericana para la
Investigación y el Desarrollo Educativo
ISSN $2007-7467$

Los restaurantes en riesgo, $71 \%$, mencionaron que ya se encontraban adaptando algunas estrategias digitales para atraer clientes, y obtener más ventas, mientras que el restante $29 \%$ comentó que estaban fuera de riesgo de cierre y que se debió a que tenían presencia en el mercado digital.

Durante la entrevista, los restauranteros comentaron que aún dos meses después de que se ordenara el cierre definitivo de los establecimientos, y se cambiara su forma de operación a solo entrega a domicilio, continuaban pensando que la situación que vivían sería momentánea y que los establecimientos que dirigían y poseían lograrían sobrevivir.

Sin embargo, con el paso del tiempo, se comenzaron a dar cuenta de que no era así, y que el cierre de establecimientos cada día se prolongaba más, un lapso de tiempo indefinido, y que trabajar bajo la nueva modalidad se hacía más difícil. Cada semana que pasaba sus ventas disminuían drásticamente.

También se les preguntó acerca del porcentaje de ventas que obtenían los restaurantes por medio de las aplicaciones food delivery antes de que se ordenara el cierre obligatorio. Estos datos se muestran en la figura 3.

Figura 3. Ventas realizadas mensualmente (en porcentaje) por medio de las aplicaciones food delivery

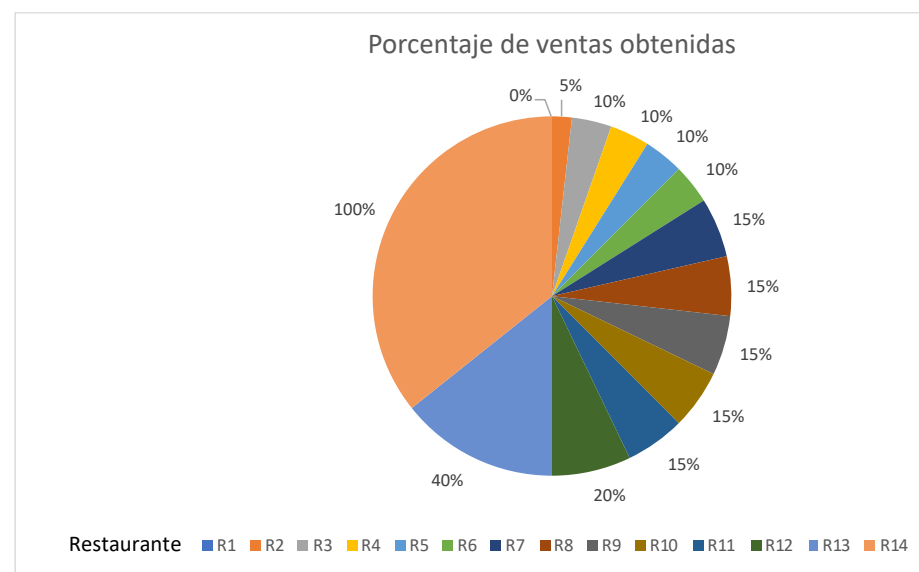

Fuente: Elaboración propia

Las respuestas apuntan que $43 \%$ de los restaurantes obtenía de $0 \%$ a $10 \%$ de ventas por aplicaciones antes de que tuvieran que cerrar al público; otro $43 \%$ mencionó que sus ventas eran de $15 \%$ a $20 \%$ a través de este canal. Cabe mencionar que los restauranteros argumentaron que las ventas por aplicaciones no eran muy comunes, y tampoco se le prestaba demasiada importancia, únicamente usaban las aplicaciones para tener presencia en el mercado. 


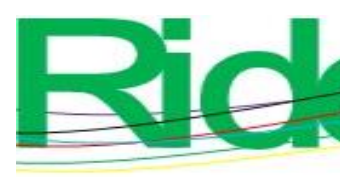

\section{Revista Iberoamericana para la Investigación y el Desarrollo Educativo ISSN 2007 - 7467}

Únicamente $7 \%$ de los restaurantes, cuyas ventas eran mayormente por medio de aplicaciones, obtuvo ventas de $40 \%$. Y en el caso particular de un restaurante que operaba únicamente por medio de aplicaciones, sus ventas no se vieron afectadas y seguían siendo de $100 \%$.

Después de analizar la información obtenida, se observó que las ventas que se obtenían por medio de aplicaciones de food delivery eran en su mayoría poco significativas. Los restauranteros y gerentes entrevistados mencionaron que, si bien se les brindaba atención a las ventas por aplicaciones, no eran su objetivo principal, eran herramientas auxiliares, su atención se centraba principalmente en las ventas físicas para el consumo dentro del establecimiento. Pero, con los eventos ya tratados, se vieron en la necesidad de realizar modificaciones y adaptaciones al tipo de servicio brindado para continuar en el mercado.

En la entrevista también se preguntó por el porcentaje de crecimiento en ventas que los establecimientos pudieron obtener por medio del uso de aplicaciones food delivery después de que se ordenara el cierre de los restaurantes al público y la entrega "solo a domicilio". La figura 4 muestra la comparativa entre las ventas (en porcentaje) que obtenían los restaurantes por medio de estas aplicaciones antes y después del cierre obligatorio.

Figura 4. Comparativa entre las ventas (en porcentaje) de las food delivery apps

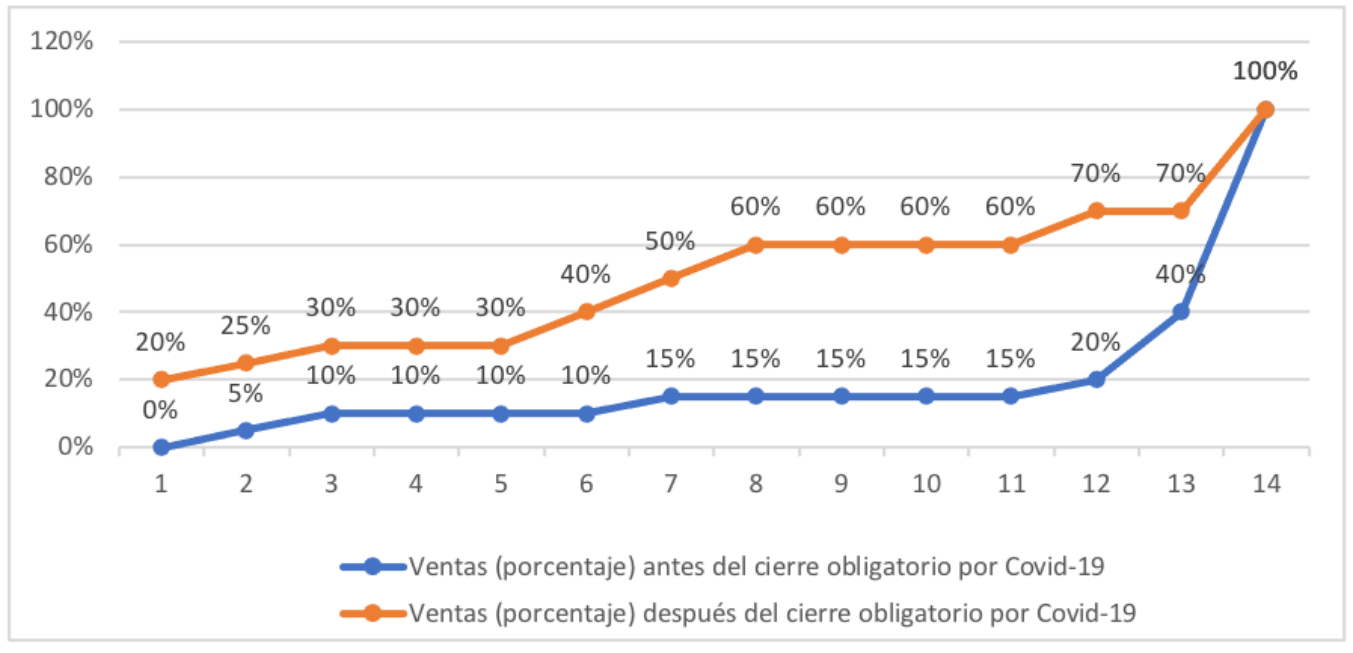

Fuente: Elaboración propia

Como se puede apreciar, $43 \%$ de los restaurantes entrevistados presentó un aumento de entre $20 \%$ y $40 \%$ en sus ventas. Asimismo, otro $43 \%$ de los restaurantes presentó un aumento importante entre $50 \%$ y $70 \%$ de ventas por aplicaciones después de que iniciara la operación bajo la nueva modalidad; mientras que el $14 \%$ restante obtuvo un aumento muy considerable entre $80 \%$ y $100 \%$. Todas estas cifras muestran un aumento en las ventas 


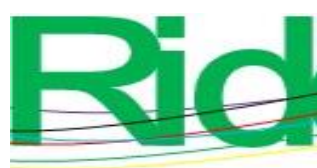

Revista Iberoamericana para la Investigación y el Desarrollo Educativo ISSN $2007-7467$

obtenidas por las aplicaciones food delivery después del cierre de establecimientos debido a la pandemia.

Asimismo, se encuestó a los restauranteros acerca de las características que debe tener una aplicación food delivery, las cuales se muestran en la tabla 2.

Tabla 2. Características que debe tener una aplicación food delivery

\begin{tabular}{|c|r|}
\hline Porcentaje de restaurantes & \multicolumn{1}{|c|}{ Observaciones mencionadas } \\
\hline $14 \%$ & $\bullet$ Diseño atractivo \\
& - Fácil de acceder desde cualquier dispositivo \\
& - Diversificación de productos \\
\hline $58 \%$ & - Fácil uso \\
\hline $14 \%$ & - Fotos llamativas y publicidad \\
& - Comisiones accesibles \\
& - Promociones \\
\hline $14 \%$ & $\bullet$ Popularidad \\
\hline
\end{tabular}

Fuente: Elaboración propia

Así, $14 \%$ de los restauranteros considera que una aplicación debe tener un buen diseño, contar con carga rápida, además de tener una gran variedad de productos; $58 \%$ considera que la aplicación debe ser fácil de usar tanto para el restaurante como para los clientes; $14 \%$ mencionó que es de gran importancia incluir fotos atractivas, que las comisiones deben ser accesibles y contener promociones; $14 \%$ mencionó que también es importante decidir cuál aplicación usar y tomar en cuenta la popularidad de estas. Durante la entrevista, los restauranteros mencionaron que debido al uso de aplicaciones han podido expandir y diversificar su mercado y acercarse a un público más joven y con más exigencias.

Los restaurantes entrevistados usaban en promedio tres aplicaciones cada uno. Las más usadas fueron Uber Eats, Rappi y Sin Delantal; las menos usadas fueron Didi Food y Postmates. Cabe mencionar que hubo un restaurante que operaba específicamente por Facebook y WhatsApp.

Aunado a ello, $36 \%$ de la muestra mencionó que es posible seguir la operación bajo esta modalidad, sin embargo, también se mencionó que para que el restaurante pueda seguir operando bajo la modalidad de solo a domicilio a largo plazo y que además sea rentable, se tiene que modificar el negocio, hacer adaptaciones en cuanto a la estructura organizacional, 


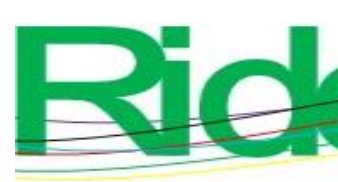

Revista Iberoamericana para la Investigación y el Desarrollo Educativo ISSN $2007-7467$

reducir el personal de servicio, aumentar la capacidad de producción y fuerte uso de food delivery apps.

Por otra parte, $36 \%$ de ellos mencionó que no ve posible continuar con esta modalidad de operación por un tiempo prolongado, que, de seguir así, tendrían que cerrar sus puertas de forma definitiva; mientras $29 \%$ dijo que sí lo ve posible. Cabe mencionar que estos restaurantes son más sólidos y llevan más tiempo de operación, por lo que podrían evolucionar a la nueva modalidad de operación sin peligro de cierre.

En cuanto a los inconvenientes que los empresarios mencionaron acerca del uso de aplicaciones de food delivery, $21 \%$ considera que las comisiones son un punto importante para analizar antes de comenzar a usar una aplicación, ya que hay algunas que cobran comisiones muy altas, por lo que recomiendan hacer una comparación antes de elegir para que sea rentable. Asimismo, $7 \%$ menciona que el único problema que ha tenido usando aplicaciones es que a veces los repartidores cometen errores con las entregas, lo que compromete la imagen del restaurante y genera costos para el restaurante (algunos deciden tener sus propios repartidores dados de alta en las aplicaciones para evitar este tipo de eventos). Y $29 \%$ no mencionó inconveniente alguno, comentaron que se encuentran trabajando de forma óptima. Cabe mencionar que son los establecimientos que más tiempo llevan en el mercado, se encuentran bien posicionados y tienen ventas altas, aun en época de pandemia. Finalmente, 7 \% no usa aplicaciones food delivery, se anuncian por redes sociales, por lo que se encargan de realizar los envíos con personal del propio establecimiento, y $36 \%$ de los entrevistados menciona que en ocasiones la demanda es tan alta que las aplicaciones se saturan, generan atrasos y pérdidas monetarias.

Para los establecimientos, el uso de aplicaciones ha sido una estrategia viable para continuar en operación, ya que son herramientas populares que funcionan para captar clientes, expandir el mercado, obtener popularidad sin pagar demasiado por una campaña publicitaria, se obtienen ventas, influyen de manera importante en la adaptación y mejora de los productos, se conservan empleos y el establecimiento sigue en operación ante este nuevo panorama que compromete el futuro de las MIPyMES restauranteras de la Ciudad de México.

Después de realizar el análisis de los datos obtenidos, se observó que el uso de las aplicaciones food delivery está totalmente relacionada con la capacidad de continuar en operación para los restaurantes, debido a que, además de ofrecer una alternativa para continuar generando ventas, influye en el acercamiento a más clientes y a diversos públicos y se reduce la plantilla de personal en áreas de servicio, como lo mencionaron algunos 


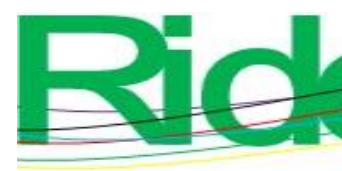

Revista Iberoamericana para la
Investigación y el Desarrollo Educativo
ISSN $2007-7467$

restaurantes. Pero también es importante tomar en cuenta que el uso de estas aplicaciones implica supervisar que los repartidores entreguen de forma correcta el paquete, supervisar pedidos y evitar la saturación para no llegar a la sobreventa, mencionaron también los restauranteros.

\section{Discusión}

Los datos obtenidos reflejaron que los restaurantes se encontraban en momentos de incertidumbre por el riesgo de cerrar de forma permanente ante el hecho de tener que continuar operando bajo la nueva modalidad. Sin embargo, encontraron en el uso de aplicaciones food delivery una estrategia alterna para continuar operando. Los datos obtenidos muestran que hubo una disminución en sus ventas, pero, aun así, podían seguir trabajando.

Mantener la operación del establecimiento fue lo más importante para los restauranteros, generar ventas que alcanzaran para costear el pago de salarios y servicios, además de seguir ofreciendo productos con seguridad y calidad a los comensales. Al respecto, Kim et al. (2019) señalan que es obligación de los restauranteros asegurar que exista una buena relación entre restaurante y cliente, y que es de vital importancia cuidarla para fomentar la creación de valor para los involucrados. El uso de estas herramientas y el cuidado de su contenido ayudó a los restaurantes a posicionarse dentro de la nueva forma de competencia y de ventas exclusivamente por aplicaciones; también ayudó a mantener clientes y aumentar sus clientes potenciales, y con esto su estatus en el mercado.

Los restauranteros mencionaron que es de vital importancia que la aplicación sea fácil de usar, contenga promociones, sea accesible respecto a las formas de pago y genere una sensación de bienestar y confianza en los clientes. En este sentido, Arellano y Acosta (2020) sugieren, para apoyar a los restaurantes, una alianza entre el Gobierno y los empresarios para crear nuevas aplicaciones que sean de uso libre, y que no causen gastos económicos excesivos a los restaurantes. Esta iniciativa sería adecuada durante esta época de pandemia. Sin duda se aumentaría la permanencia en el mercado de los establecimientos.

Mitali (2019) explica que al hacer una entrega de alimentos satisfactoria se fomenta la fidelidad de clientes a los restaurantes, y se genera promoción para llegar a nuevos clientes y generar más ventas. Los restaurantes de este estudio, al hacer uso de las aplicaciones, generaban ventas, y conocían a clientes nuevos y clientes habituales, además de que les servía como estrategia para desarrollar nuevos productos. Los restauranteros creen que cuando todo 


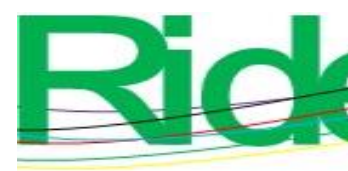

Revista Iberoamericana para la
Investigación y el Desarrollo Educativo
ISSN $2007-7467$

vuelva a la normalidad los clientes podrán tener un servicio más personalizado, lo que puede generar mayores ganancias para ellos.

Los resultados de esta investigación también concuerdan con los hallazgos de He et al. (2019), quienes mencionan que las aplicaciones de este tipo brindan beneficios no solo a los clientes, quienes pueden adquirir alimentos sin tener que salir del lugar en el que se encuentren, a buen precio, calidad, rapidez y con diferentes formas de realizar el pago; también representa un beneficio para los restaurantes, ya que generan ventas en línea sin tener que ampliar la capacidad de aforo, contratar a más personal o adecuar las instalaciones, por lo que, también, la utilidad aumenta.

\section{Conclusiones}

Los restaurantes consideran que antes de usar aplicaciones food delivery es importante analizar las opciones disponibles en el mercado, hacer una comparativa de costos de comisión, diseño y facilidad de uso tanto para los restaurantes como para los clientes. Además, es útil tomar en cuenta que no se saturen las aplicaciones, lo cual genera sobreventas y, por extensión, mala reputación para el establecimiento.

Después de analizar los datos obtenidos, se observó que el uso de las aplicaciones food delivery en las MIPyMES restauranteras de la Ciudad de México ha funcionado como estrategia de ventas, lo que les permitió seguir en operación para pagar salarios y servicios.

$\mathrm{Y}$ aunque varios restaurantes ya contaban con un medio digital como forma de comunicación con sus clientes, ya sea por medio de las aplicaciones en cuestión o por redes sociales, solo lo usaban como forma de presencia en el mercado y no como medio de obtener ventas, por lo que el proceso de adaptación y cambio al que se sometieron tuvo que ser rápido y no libre de dificultades. Y aunque el proceso fue arduo, las aplicaciones food delivery han funcionado como medio para solventar los gastos esenciales de los restaurantes y continuar con su operación.

\section{Futuras líneas de investigación}

Los resultados de esta investigación evidenciaron que existen otros factores involucrados para que los empresarios restauranteros quieran adoptar y usar las food delivery apps. Por lo tanto, investigar cuáles son estos factores y el impacto que tienen en su adopción sería el siguiente paso luego de este trabajo. Asimismo, los hallazgos mostraron que para que 


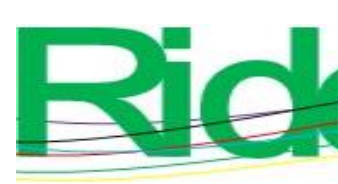

Revista Iberoamericana para la
Investigación y el Desarrollo Educativo
ISSN $2007-7467$

un restaurante continúe operando con las aplicaciones digitales requiere de hacer modificaciones en su modelo de negocio, lo cual es una oportunidad para realizar una investigación futura acerca de modelos de negocio innovadores para la industria restaurantera.

\section{Agradecimientos}

Al Instituto Politécnico Nacional, al Consejo Nacional de Ciencia y Tecnología y a los restaurantes que accedieron a formar parte de esta investigación.

\section{Referencias}

Arellano, R. y Acosta, E. (2020). Uso de apps delivery service en microempresas gastronómicas de reciente creación en la Ciudad de México. Administración y Organizaciones, 23(44), 35-54. Recuperado de https://doi.org/https://doi.org/10.24275//uam/xoc/dcsh/rayo/2020v23n44/Arellano.

Asociación Mexicana de Restaurantes [AMR]. (4 de enero de 2019). Datos de la industria. Cifras de la industria restaurantera. Recuperado de https://www.amr.org.mx/datosde-la-industria.phtml

Asociación Mexicana de Restaurantes [AMR]. (24 de abril de 2020). 70\% de los restaurantes en riesgo de desaparecer. Recuperado de http://www.amr.org.mx/noticias.phtml?id=3508\&categoria=NOTICIAS.

Cámara Nacional de la Industria de Productos Alimenticios Condimentados [CANIRAC]. (2015). Todo sobre la mesa. Dimensiones de la Industria Restaurantera en México.

Centro Nacional de Programas Preventivos y Control de Enfermedades [CENAPRECE]. (2018). Plan nacional para la preparación y respuesta ante la intensificación de la influenza estacional o ante una pandemia de influenza. México: Secretaría de Salud. Recuperado de https://www.gob.mx/cms/uploads/attachment/file/356290/Plan_Nacional_Influenza. pdf.

Cho, M., Bonn, M. A. and Li, J. (2019). Differences in perceptions about food delivery apps between single-person and multi-person households. International Journal of Hospitality Management, 77, 108-116. Retrieved from https://doi.org/https://doi.org/10.1016/j.ijhm.2018.06.019. 


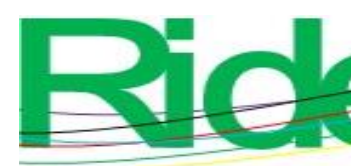

Revista Iberoamericana para la Investigación y el Desarrollo Educativo ISSN $2007-7467$

Chung, H. S., Choi, S. E. and Choi, D. K. (2017). The effect of perceived risk, perceived benefits on purchase intention for delivery food. Korean Journal of Hospitality \& Tourism, 26(8), 71-86.

Escamilla, O. (8 de abril de 2020). El camino que deben seguir las delivery apps para ganarse al consumidor. Merca2.0. Recuperado de https://www.merca20.com/el-camino-quedeben-seguir-las-delivery-apps-para-conquistar-a-los-consumidores/

Fernández, C. (2020). Impacto en el mercado laboral de las medidas de aislamiento para combatir el COVID-19. Recuperado de http://hdl.handle.net/11445/3935.

Fernández, F. (26 de marzo de 2020). Sirviendo a México. Nota informativa. Recuperado el 19 de octubre de 2021 de: https://canirac.org.mx/articulos/index.php?id=1516.

Forbes Staff. (19 de marzo de 2020). Uber Eats apoyará a pymes al no cobrar los pedidos por coronavirus. Forbes México. Recuperado el 26 de octubre de 2021 de: https://www.forbes.com.mx/negocios-uber-eats-apoyara-pequenas-medianasempresas-cobrar-pedidos/.

Flores, L. (7 de agosto de 2018). 6 apps móviles que ofrecen comida a domicilio. Food \& Wine. Recuperado de https://foodandwineespanol.com/6-apps-de-comida-quepuedes-descargar/.

Galeano, S. (5 de marzo de 2019). Uber eats: líder entre las apps de comida a domicilio en México. Marketing4EcommerceMX. Recuperado de https://marketing4ecommerce.mx/uber-eats-lider-entre-las-apps-de-comida-adomicilio-en-mexico/.

Giles, C. A. (2020). Recomendaciones para las MIPyMES. ¿Qué hacer para sobrevivir a la pandemia del COVID-19? NotasEstratégicas, (86), 1-13. Recuperado de http://bibliodigitalibd.senado.gob.mx/handle/123456789/4845.

He, Z., Han, G., Cheng, T. C. E., Fan, B. and Dong, J. (2019). Evolutionary food quality and location strategies for restaurants in competitive online-to-offline food ordering and delivery markets: An agent-based approach. International Journal of Production Economics, 215, 61-72. Retrieved from https://doi.org/10.1016/j.ijpe.2018.05.008.

Hernández, R. y Mendoza, C. (2018). Metodología de la investigación. Las rutas cuantitativa, cualitativa y mixta (1. ${ }^{\mathrm{a}}$ ed.). Ciudad de México, México: McGraw-Hill. Instituto Nacional de Estadística y Geografía [INEGI]. (2019). Censos económicos 2019. La industria restaurantera en México. Aguascalientes, México: Instituto Nacional de $\begin{array}{llll}\text { Estadística } & \text { y } & \text { Geografía. } & \text { Recuperado de }\end{array}$ 


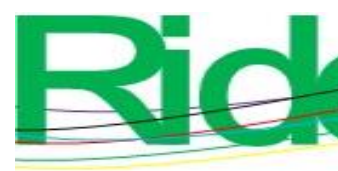

Revista Iberoamericana para la
Investigación y el Desarrollo Educativo
ISSN $2007-7467$

https://www.inegi.org.mx/contenidos/productos/prod_serv/contenidos/espanol/bvine gi/productos/nueva_estruc/702825199357.pdf.

Kim, E., Tang, L. y Bosselman, R. (2019). Customer Perceptions of Innovativeness: An Accelerator for Value Co-Creation. Journal of Hospitality \& Tourism Research, 43(6), 807-838. Retrieved from https://doi.org/10.1177/1096348019836273.

Konfío. (2020a). El impacto del COVID-19 en el sector comercio en México. Recuperado de https://konfio.mx/tips/articulos-especiales/impacto-del-coronavirus-en-el-sectorcomercial-de-mexico/.

Konfío. (2020b). El deterioro de la industria restaurantera en México. Recuperado de https://konfio.mx/tips/articulos-especiales/impacto-coronavirus-sectorrestaurantero/.

Miranda, P. y Morales, A. (28 de febrero de 2020). Se confirma primer caso de coronavirus en México. El Universal. Recuperado de https://www.eluniversal.com.mx/nacion/sociedad/coronavirus-en-mexicoconfirman-primer-caso.

Mitali, G. (2019). A Study on Impact of Online Food Delivery App on Restaurant Business. Special Reference to Zomato and Swiggy. International Journal of Research and Analytical Reviews, 6(1), 889-893.

Nabor, A. (13 de mayo de 2020). Cuándo reanudarán operaciones los restaurantes en la CDMX. El Universal. Recuperado de https://www.eluniversal.com.mx/menu/cuando-reanudaran-operaciones-losrestaurantes-en-la-cdmx.

Organización de la Naciones Unidas [ONU]. (16 de marzo de 2020). El coronavirus se puede parar: China lo ha demostrado. Noticias ONU. Recuperado de https://news.un.org/es/interview/2020/03/1471242.

Organización Mundial de la Salud [OMS]. (11 de octubre de 2018). Cólera: Alertas y actualizaciones epidemiológicas. Alerta y respuesta mundiales. Recuperadao de https://www3.paho.org/hq/index.php?option=com_topics\&view=rdmore \&cid=2160 $\&$ Itemid=40745+\&lang=es

Organización Mundial de la Salud [OMS]. (25 de septiembre de 2017). ZikaEpidemiological Report Mexico. Recuperado de https://www.paho.org/hq/dmdocuments/2017/2017-phe-zika-situation-reportmex.pdf. 


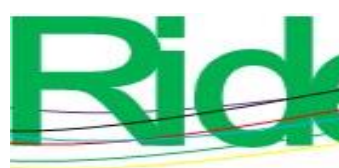

Revista Iberoamericana para la
Investigación y el Desarrollo Educativo
ISSN $2007-7467$

Organización Mundial de la Salud [OMS]. (16 de abril de 2019). Alerta Epidemiológica

Brotes por microorganismos resistentes relacionados con el turismo médico. Recuperado de https://www.paho.org/es/documentos/16-abril-2019-alertaepidemiologica-sobre-brotes-por-microorganismos-resistentes

Organización Mundial de la Salud [OMS]. (11 de marzo de 2020). Alocución de apertura del Director General de la OMS en la rueda de prensa sobre la COVID-19 celebrada el 11 de marzo de 2020. Recuperado de https://www.who.int/es/dg/speeches/detail/who-director-general-s-opening-remarksat-the-media-briefing-on-covid-19---11-march-2020.

Sarduy, Y. (2007). El análisis de información y las investigaciones cuantitativa y cualitativa. Revista Cubana de Salud Pública, 33(3), 1-11. Recuperado de https://doi.org/10.1590/s0864-34662007000300020.

Secretaría de Salud [SS]. (12 de marzo de 2020). 086. México permanece en fase uno por COVID-19. Boletín de prensa. Recuperado de https://www.gob.mx/salud/prensa/086-mexico-permanece-en-fase-uno-por-covid-19 Secretaría de Salud [SS]. (24 de marzo de 2020). 095. Inicia fase 2 por coronavirus COVID19. Boletín de prensa. Recuperado de https://www.gob.mx/salud/prensa/095-iniciafase-2-por-coronavirus-covid-19.

Secretaría de Salud [SS]. (21 de abril de 2020). 110. Inicia la fase 3 por COVID-19. Boletín de prensa. Recuperado de https://www.gob.mx/salud/prensa/110-inicia-la-fase-3por-covid-19.

Statista. (2020). Online Food Delivery. Retrieved from https://www.statista.com/outlook/374/116/online-food-delivery/mexico\#marketglobalRevenue.

Ugalde, N. y Balbastre, F. (2013). Investigación cuantitativa e investigación cualitativa: buscando las ventajas de las diferentes metodologías de investigación. Revista de Ciencias Económicas, 31(2), 179-187. Recuperado de https://dialnet.unirioja.es/servlet/articulo?codigo $=4512073$.

Universidad Nacional Autónoma de México [UNAM]. (2020). Plataforma de información geográfica de la UNAM sobre COVID-19 en México. Recuperado de https://covid19.ciga.unam.mx/.

Yin, R. (2003). Case Study Research. Desing and Methods (5 ${ }^{\text {th }}$ ed.). United States: SAGE Publications. 


\begin{tabular}{|c|c|}
\hline Rol de Contribución & Autor (es) \\
\hline Conceptualización & $\begin{array}{l}\text { Elizabeth Acosta-Gonzaga - principal } \\
\text { Juan Manuel Sandoval-Damián - que apoya }\end{array}$ \\
\hline Metodología & $\begin{array}{l}\text { Elizabeth Acosta-Gonzaga - principal } \\
\text { Juan Manuel Sandoval-Damián - que apoya }\end{array}$ \\
\hline Software & No aplica \\
\hline Validación & $\begin{array}{l}\text { Juan Manuel Sandoval-Damián - igual } \\
\text { Elizabeth Acosta-Gonzaga - igual } \\
\text { José Leonardo Serralde-Coloapa - igual }\end{array}$ \\
\hline Análisis Formal & $\begin{array}{l}\text { Juan Manuel Sandoval-Damián - igual } \\
\text { Elizabeth Acosta-Gonzaga - principal } \\
\text { José Leonardo Serralde-Coloapa - igual }\end{array}$ \\
\hline Investigación & $\begin{array}{l}\text { Juan Manuel Sandoval-Damián - principal } \\
\text { Elizabeth Acosta-Gonzaga - igual } \\
\text { José Leonardo Serralde-Coloapa - igual }\end{array}$ \\
\hline Recursos & $\begin{array}{l}\text { Juan Manuel Sandoval-Damián - igual } \\
\text { Elizabeth Acosta-Gonzaga - igual } \\
\text { José Leonardo Serralde-Coloapa - igual }\end{array}$ \\
\hline Curación de datos & $\begin{array}{l}\text { Juan Manuel Sandoval-Damián - igual } \\
\text { Elizabeth Acosta-Gonzaga - principal } \\
\text { José Leonardo Serralde-Coloapa - igual }\end{array}$ \\
\hline $\begin{array}{l}\text { Escritura - Preparación del } \\
\text { borrador original }\end{array}$ & $\begin{array}{l}\text { Juan Manuel Sandoval-Damián - principal } \\
\text { Elizabeth Acosta-Gonzaga - igual } \\
\text { José Leonardo Serralde-Coloapa - igual }\end{array}$ \\
\hline Escritura - Revisión y edición & $\begin{array}{l}\text { Juan Manuel Sandoval-Damián - igual } \\
\text { Elizabeth Acosta-Gonzaga - igual }\end{array}$ \\
\hline Visualización & $\begin{array}{l}\text { Juan Manuel Sandoval-Damián - principal } \\
\text { Elizabeth Acosta-Gonzaga - que apoya }\end{array}$ \\
\hline Supervisión & Elizabeth Acosta-Gonzaga \\
\hline Administración de Proyectos & José Leonardo Serralde-Coloapa \\
\hline Adquisición de fondos & No aplica \\
\hline
\end{tabular}




\section{Anexo}

\section{Guía de entrevista}

La presente entrevista tiene como objetivo indagar sobre el uso de plataformas de food delivery en las MIPyMES restauranteras de la CDMX y cómo les han servido para sobrevivir al confinamiento causado por el SARS-CoV-2. Las preguntas a contestar son las siguientes:

- ¿En qué porcentaje se redujeron las ventas después de que se ordenara el cierre de establecimientos al público y se dictaminara la entrega "solo a domicilio"?

- ¿Actualmente el restaurante se encuentra en peligro de cierre permanente?

- De las ventas estimadas mensualmente antes de que se ordenara el cierre de restaurantes, ¿qué porcentaje de ventas se adquirían por medio de las aplicaciones de food delivery?

- ¿Considera que ha crecido, y cuál es el porcentaje de ventas por aplicaciones de food delivery durante la pandemia?

- ¿Cuántas y cuáles plataformas de food delivery usa el establecimiento?

- ¿ ¿Cuáles considera que son las características de la plataforma que más ventas genera al establecimiento?

- ¿ ¿Considera que el restaurante podría seguir siendo rentable bajo la modalidad de envío a domicilio?

- ¿ ¿Ha detectado algún inconveniente con el uso de las aplicaciones food delivery durante esta pandemia?

- $\quad$ En sus propias palabras, cómo diría que le benefició el uso de las aplicaciones de food delivery: 